\section{Navigated intraoperative ultrasonography for brain tumors: a pictorial essay on the technique, its utility, and its benefits in neuro-oncology}

\author{
Ujwal Yeole, Vikas Singh, Ajit Mishra, Salman Shaikh, Prakash Shetty, Aliasgar Moiyadi \\ Neurosurgery Services, Department of Surgical Oncology, Tata Memorial Centre and Homi \\ Bhabha National Institute, Mumbai, India
}

Intraoperative imaging has become one of the most important adjuncts in neurosurgery, especially in the surgical treatment of intra-axial tumors. Navigation and intraoperative magnetic resonance imaging have limitations, and intraoperative ultrasonography (IOUS) has emerged as a versatile and multifaceted alternative. With technological advances in ultrasound scanners and newer multifunctional probes, the potential of IOUS is increasingly being utilized in the resection of tumors. The addition of image guidance to IOUS has exponentially increased the power of this technique. Navigated ultrasonography (nUS) can now overcome many of the limitations of conventional standalone two-dimensional ultrasonography. In this pictorial essay, we outline our nUS technique (both two- and three-dimensional) for the resection of intra-axial tumors with illustrated examples highlighting the various steps and corresponding benefits of the technique.

Keywords: Intraoperative ultrasound; Navigated ultrasound; Brain neoplasms; Intraoperative imaging

\section{Introduction}

Up-to-date intraoperative imaging has become an important component of most surgical treatments for brain tumors. Conventional image-guided surgery (or neuronavigation), which relies on computed tomographic (CT) or magnetic resonance (MR) images that are acquired preoperatively, is limited by the deformation (brain shift) that occurs once the dura is opened and the brain is manipulated, rendering such preoperatively-acquired "maps" inaccurate and unusable [1]. This has led to the proliferation of intraoperative imaging tools to provide updated, or corrected, images. Intraoperative $\mathrm{CT}$ is not very useful due to its inherent lack of soft-tissue resolution and the associated radiation exposure. Intraoperative MR (IOMR) has been regarded as the gold standard in this context, but it remains a logistically and financially unattractive option in most neurosurgical settings. Intraoperative ultrasonography (IOUS) has proven a useful, easily available, and cost-effective tool for neurooncological procedures, overcoming the limitations posed by IOMR [2,3]. Standard two-dimensional (2D) grey-scale ultrasonography (US) provides rapid, repeated, real-time intraoperative updates without altering the surgical workflow. Advances in US technology have led to the development of

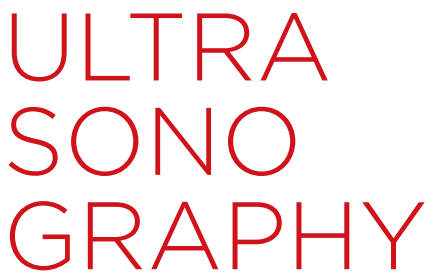

PICTORIAL ESSAY

https://doi.org/10.14366/usg.20044 pISSN: 2288-5919 e elSSN: 2288-5943 Ultrasonography 2020;39:394-406

Received: March 21, 2020

Revised: May 14, 2020

Accepted: June 17, 2020

Correspondence to:

Aliasgar Moiyadi, MCh, Neurosurgery Services, Department of Surgical Oncology, Tata Memorial Centre and Homi Bhabha National Institute, Mumbai 400012, India

Tel. +91-9324696948

E-mail: aliasgar.moiyadi@gmail.com

This is an Open Access article distributed under the terms of the Creative Commons Attribution NonCommercial License (http://creativecommons.org/ licenses/by-nc/4.0/) which permits unrestricted noncommercial use, distribution, and reproduction in any medium, provided the original work is properly cited.

Copyright (C) 2020 Korean Society of Ultrasound in Medicine (KSUM)

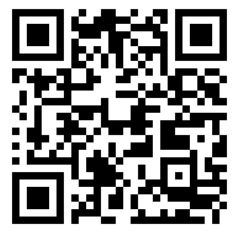

How to cite this article:

Yeole U, Singh V, Mishra A, Shaikh S, Shetty $P$, Moiyadi A. Navigated intraoperative ultrasonography for brain tumors: a pictorial essay on the technique, its utility, and its benefits in neuro-oncology. Ultrasonography. 2020 0ct;39(4):394-406. 
newer-generation scanners specifically designed for intracranial applications. With superior image quality combined with Doppler imaging and angiography as well as newer US applications such as contrast-enhanced US (CEUS) and elastosonography, IOUS has arguably become the imaging modality of choice in neurooncological surgery. With its minimal alteration of the surgical workflow (unlike MR) and ease of repeated use, IOUS is an attractive tool. That said, 2DUS does pose certain limitations, mainly related to the problems of orienting a limited field of view and interpreting planar images in unconventional planes. Navigated ultrasonography (nUS) utilizing either 2D (n2DUS) or three-dimensional (3D; n3DUS) volumes helps overcome many of the limitations of standalone 2DUS.

In this report, we outline the n3DUS technique that we use during brain tumor surgery. We describe the technical nuances and discuss, with illustrative cases, the spectrum of applications and benefits. We used a commercially available high-end ultrasound scanner (bk5000, BK Medical, Herlev, Denmark) digitally integrated with Brainlab KICK ultrasound navigation software (Brainlab AG, Munich, Germany) to perform n3DUS in 34 cases. The present paper is focused on the application of this system during brain tumor surgery. The setup, detailed insonation parameters, and operating room workflow are provided in the Supplementary Data 1, as well as in Figs. 1-3.

\section{DUS Acquisition}

After completion of the craniotomy, a curved linear probe with a small footprint $(29 \times 10 \mathrm{~mm})$ and a frequency range of $5-13 \mathrm{MHz}$ (Craniotomy N13C5 transducer, BK Medical) is introduced into the surgical field, and a wide transdural 2DUS scan of the entire exposed surface is performed (Fig. 4). Adjusting the parameters

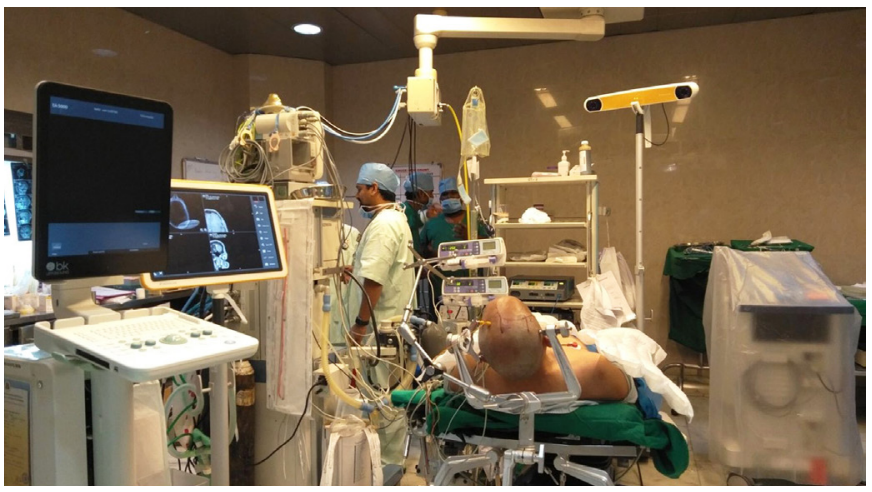

A

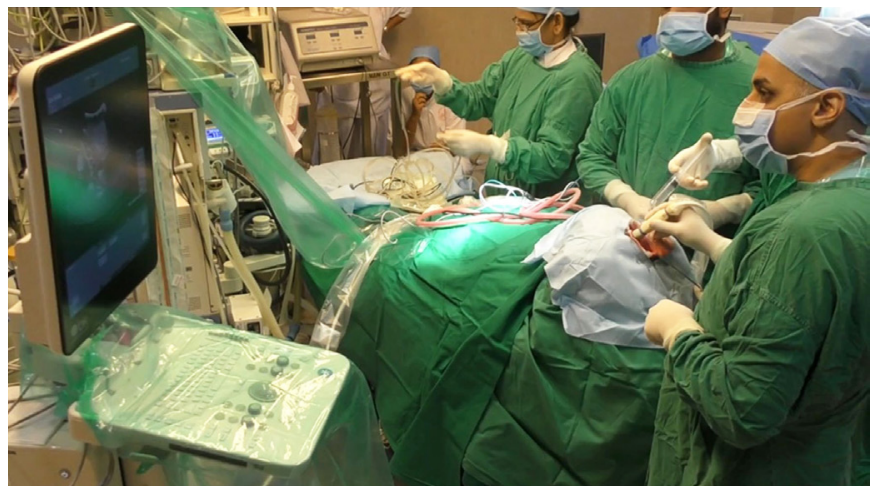

B

Fig. 1. Operation theatre layout.

A. Note the position of the ultrasound scanner in relation to the navigation system and its proximity to the operating field. B. Covering the control panel with a sterile drape allows direct control by the surgeon.

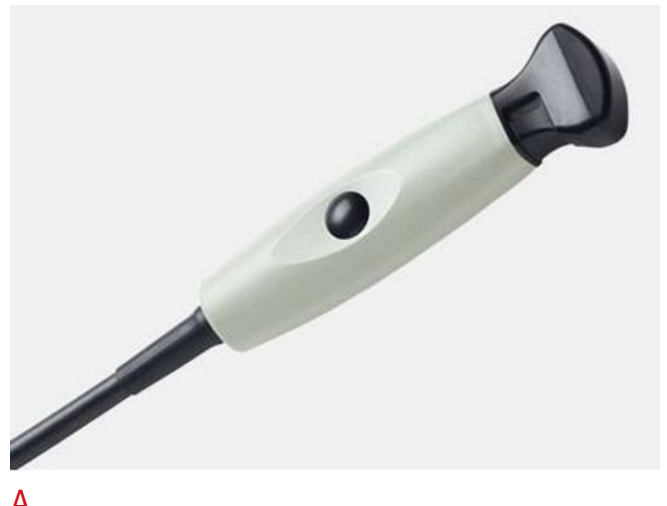

A

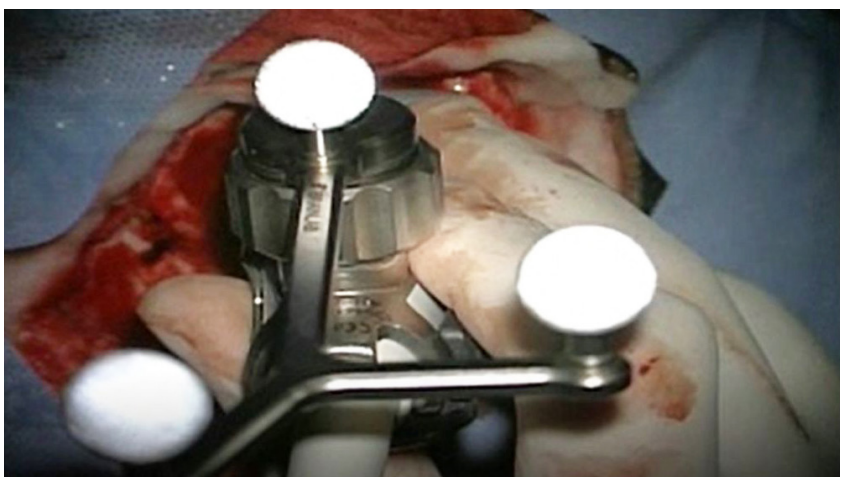

B

Fig. 2. Navigated ultrasonography (nUS).

A. A convex liner probe is used for acquiring the nUS. B. A localizer with reflective spheres is fixed in a predetermined position onto the ultrasound (US) probe. This is calibrated to the navigation system, allowing the US images to be registered in the same frame of reference as the magnetic resonance imaging scans previously registered on the navigation system. 


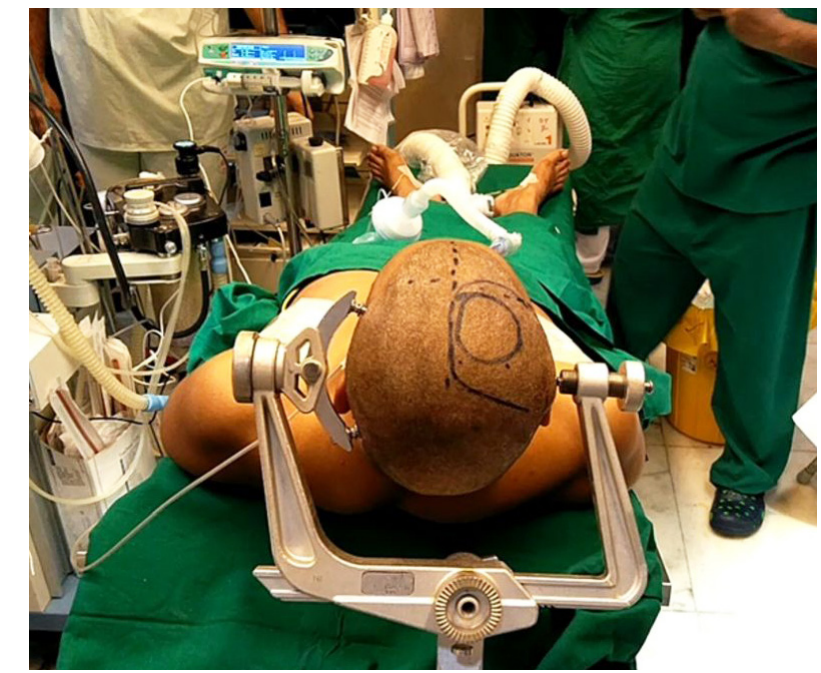

A

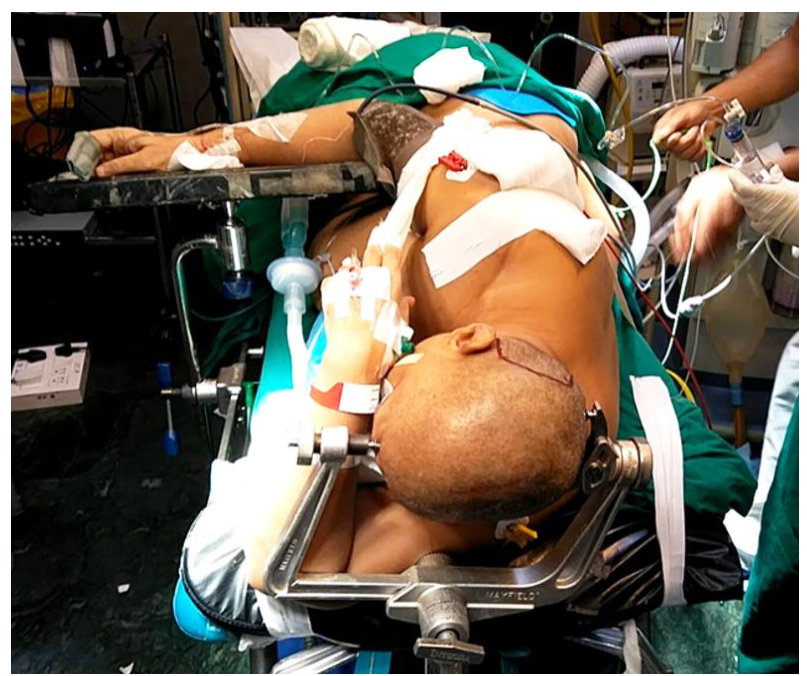

B

Fig. 3. Positioning of the patient during ultrasound (US)-guided surgery.

It is essential to plan the position in such a way that the conditions can be optimized for performing a post-resection scan, and the cavity should be able to be filled with saline to facilitate good acoustic coupling. The aim is to keep the operating surface as flat, or parallel to the floor, as possible. A right frontal craniotomy position is shown in (A) and a paramedian suboccipital cranitomy position in (B). With careful planning, an optimal US scan can be obtained for virtually any cranial exposure.

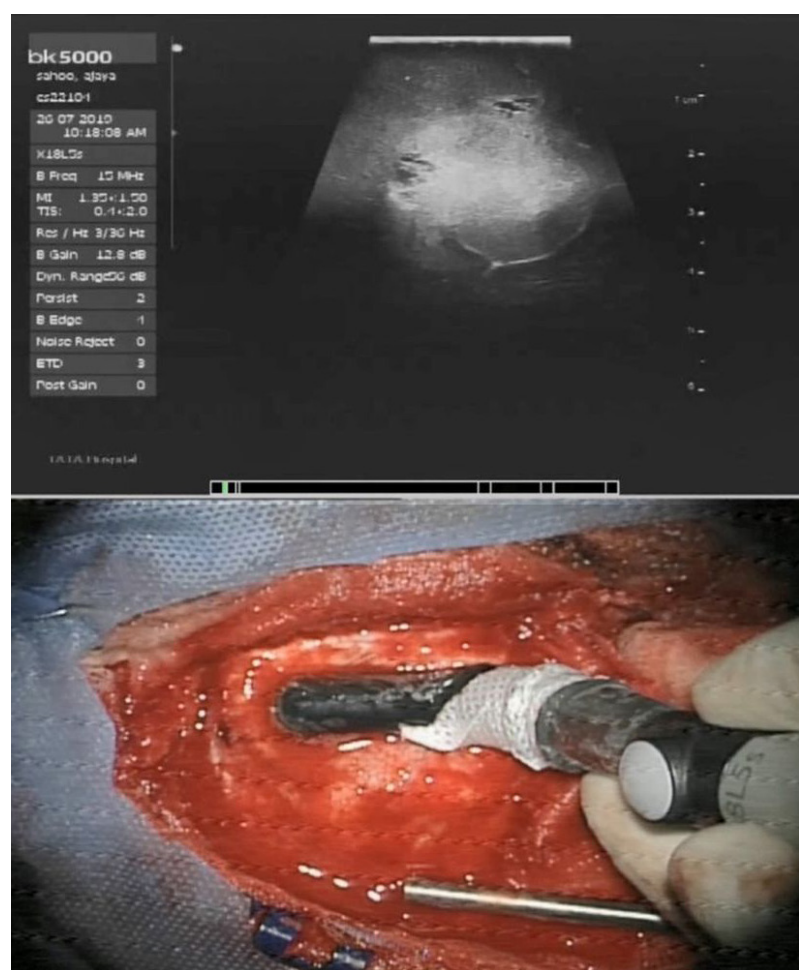

A

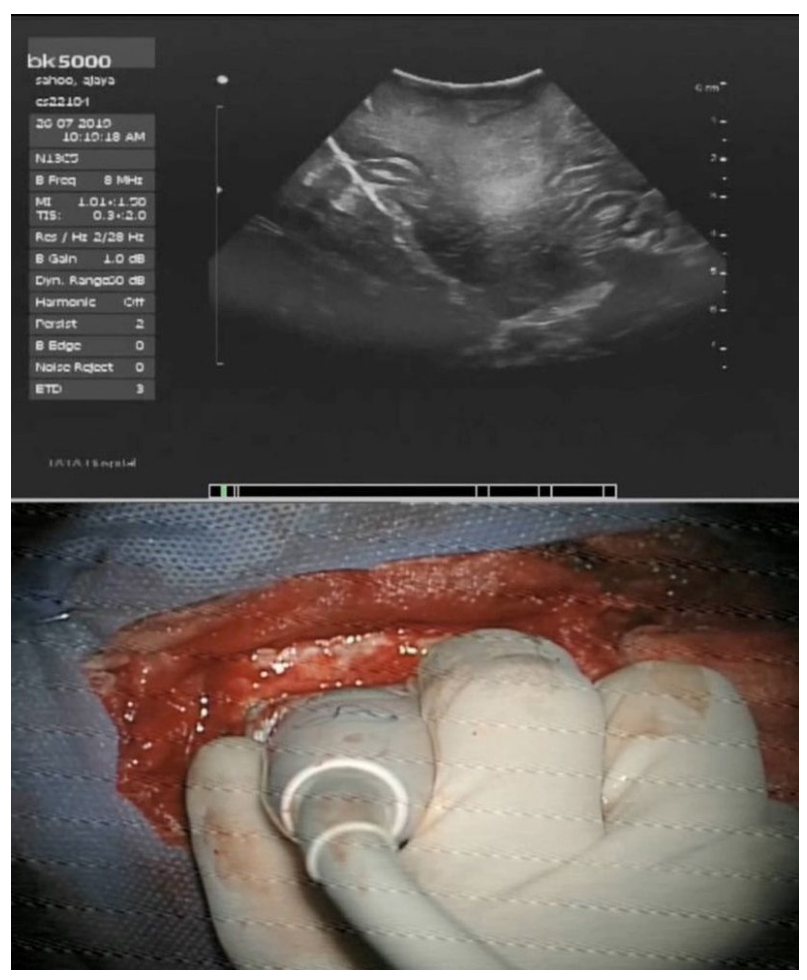

B

Fig. 4. Two-dimensional ultrasound.

The same lesion is insonated with two different probes. A. A high-frequency linear, 6- to 15-MHz hockey-stick probe is being used, providing exquisite anatomical details of a superficially-located tumor with poorer delineation of deeper structural details. B. A curvilinear, 5- to 13$\mathrm{MHz}$ probe images the same lesion, showing a panoramic view that also aids in image orientation. High-resolution images from both of the ultrasound probes are available in Supplementary Figs. 1 and 2. 
(depth, gain, and time-gain control) can help optimize the images. The US acquisition is repeated after opening the dura, as dural calcifications may interfere with optimal insonation. This image acquisition helps characterize the lesion (with regard to its extent, echogenicity, internal variability, margins, cysts, and calcific shadows) and survey the surrounding anatomical landmarks for better orientation. The falx (a linear hyperechoic structure), ventricles (anechoic and bilaterally symmetrical structures), and choroid plexus (densely hyperechoic tufts within the ventricles) are useful starting points. Performing careful orthogonal scans in two planes, preferably conventional imaging planes (axial, coronal, and sagittal; ACS), can aid in generating a 3D mental picture of the entire operative field and help the operator become oriented (Fig. 5). However, due to challenges related to the location and size of the craniotomy, it is often difficult to accomplish this with 2DUS unless one is an expert operator. At this point, additional imaging with Doppler, angiography,

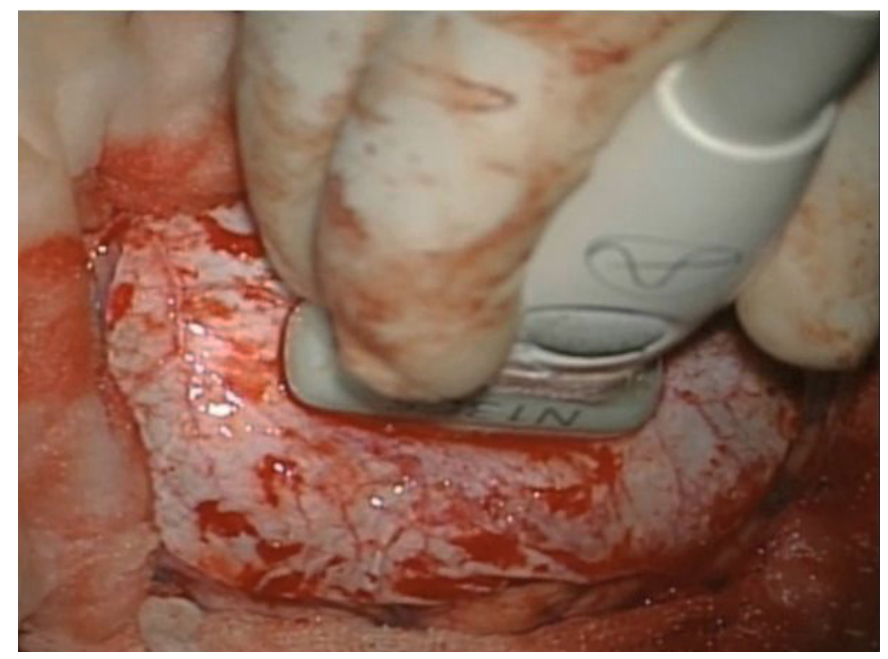

A

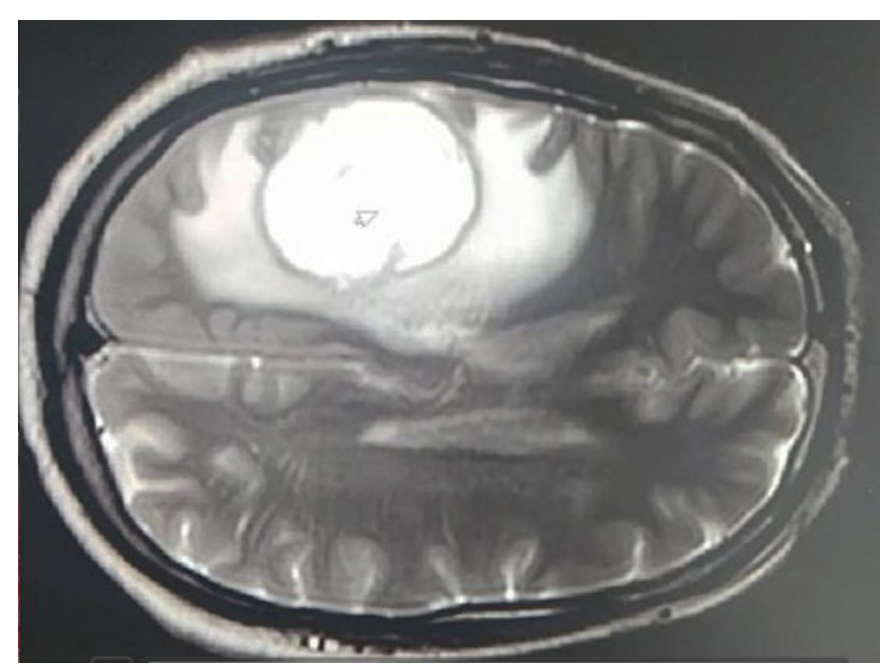

C
CEUS, or elastosonography can also be performed to further characterize the lesion if needed; however, we do not do this routinely.

\section{n2DUS}

An n2DUS scan can then be immediately acquired in the precalibrated system by simply attaching the navigation localizer to the probe (Fig. 3). As the probe is moved across the surgical field, the corresponding 2DUS is visualized in real-time on the navigation screen superimposed on the co-displayed MR scans in the corresponding plane along with the virtual representation of the probe in the surgical field (Fig. 6). This multimodal image display instantly makes it easier to understand the plane of insonation (which may not be a true anatomical plane) and helps to manually adjust and realign the US plane along a conventional ACS plane if needed (Fig. 7). Furthermore, it is easier (particularly for novices) to identify

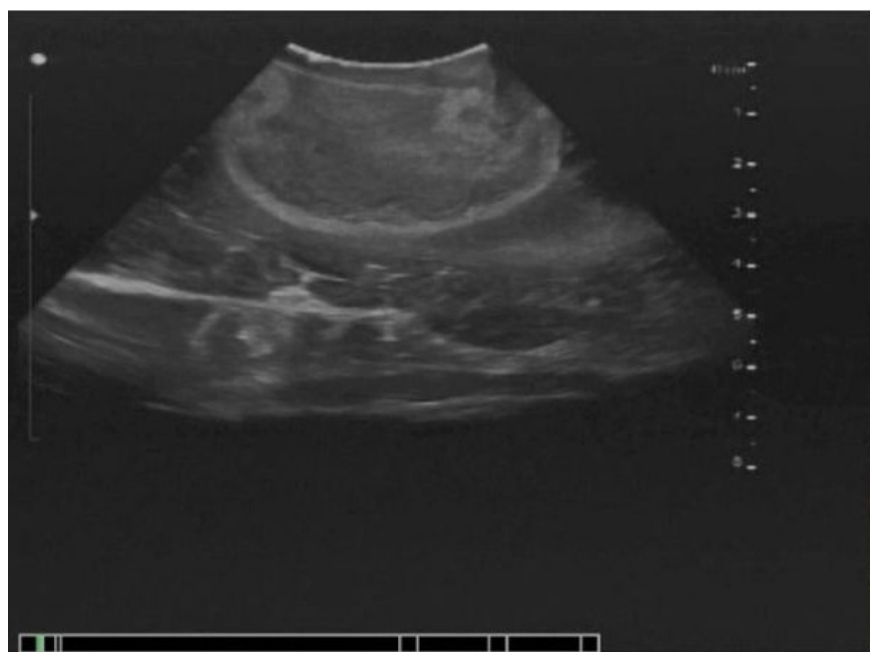

B

Fig. 5. Two-dimensional ultrasonography.

A. Probe is positioned over the surface of the dura. B. Twodimensional ultrasound image obtained in axial plane is depicted. C. Corresponding magnetic resonance image is seen in this image. It may not always be feasible to obtain such true anatomical planes, depending on the craniotomy aperture and location. 
anatomical landmarks on the US image by correlating it with the corresponding MR image in the same plane. However, multiplanar US imaging is still not possible with n2DUS unless the probe itself is rotated among the different planes. Hence, we prefer to perform 3DUS before commencing resection (Fig. 8).

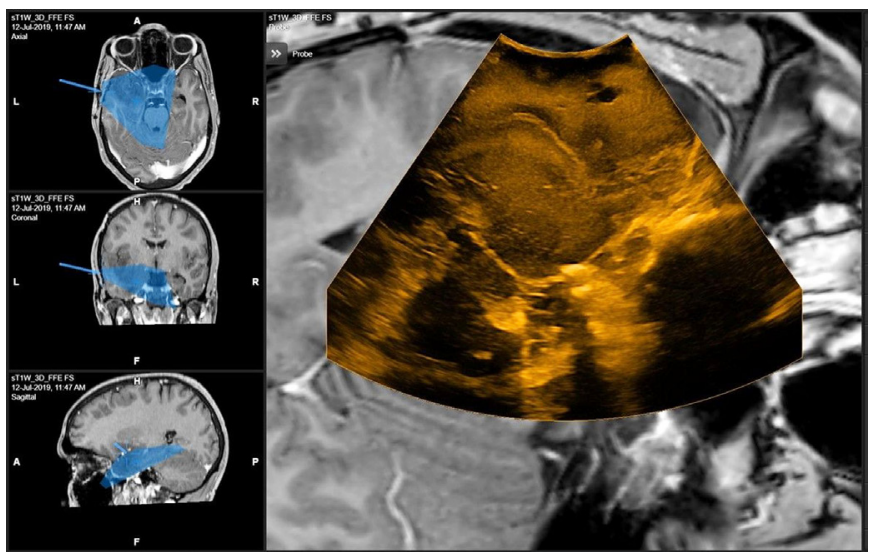

Fig. 6. Navigated two-dimensional ultrasonography (2DUS). Screenshot of the real-time navigated 2DUS from the navigation system is shown here. The right panel shows the actual ultrasound (US) image superimposed on the corresponding magnetic resonance (MR) plane. This screen is "live" and depicts the real-time moving US image as the operator sweeps the operative field, exactly duplicating the US scanner screen. The left panel shows the plane of insonation represented in the conventional axial, coronal, and sagittal MR images. Note that the plane of insonation is not a true anatomical plane, as is the case in most surgical procedures. The white dashed line outlines a tumor that appears hypointense on T1 MR and hyperechoic on US.

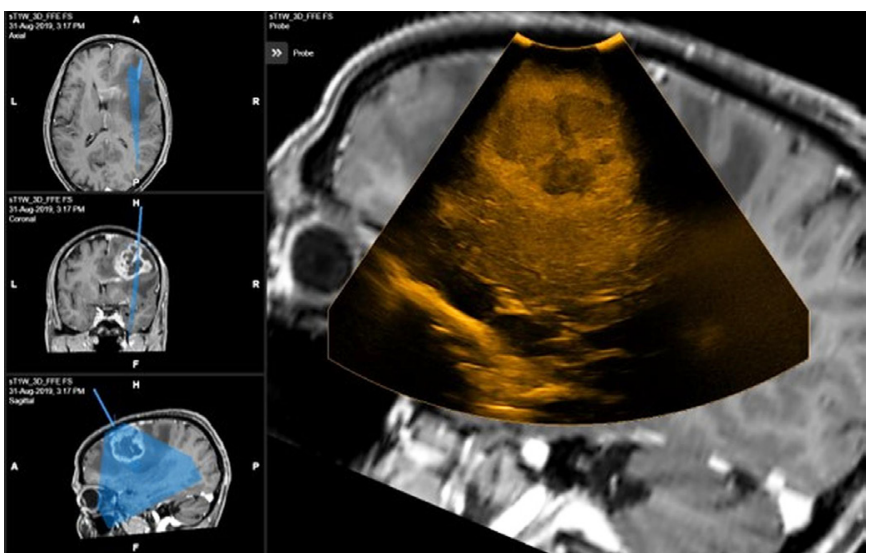

A

Fig. 7. Using navigated two-dimensional ultrasonography (2DUS) to align the real-time 2DUS image in a true anatomical plane. A. True sagittal image in a case of left frontal high-grade glioma is seen. B. True coronal image in the same case is depicted.

\section{n3DUS}

After performing live n2DUS, the probe is swept along the operative field of interest, capturing a series of 2DUS images that are reconstructed into a 3D volume (the 3D sweep). To generate as large a volume as possible, it is important to utilize the entire exposed surface. The system requires a single continuous sweep; hence, the practitioner should first use live n2DUS mode to practice a sweep that will encompass as much of the representative tumor area and surrounding brain as possible. This also ensures that no line-of-sight problems with the navigation system will be present during the actual 3DUS acquisition process. The actual sweep is then completed by slowly tilting and translating the probe over the surface of the dura (or brain) from one end of the craniotomy to the other in the chosen direction. Continuous feedback from the live n2DUS screen provides complete control over the quality and content of this 3D acquisition. Within 20 to 30 seconds, the system computes the $3 \mathrm{D}$ volume. Once this is done, the probe is set aside; then, using the navigation probe, the US image depicted on the navigation screen in multiplanar ACS views (co-displayed with the MR image) can be used for navigation (Fig. 8). This 3D US-MR image fusion technique greatly improves the ease of orientation and interpretation of US images and compensates for the limited field of view of the US system. The MR image also provides additional functional information (such as eloquent cortical areas and tracts) that can be segmented and superimposed on the US image that is subsequently acquired, thus providing the crucial functional information lacking in US imaging (Fig. 9). One problem with using preoperative MRbased navigation is the loss of accuracy that results once the dura is opened and cerebrospinal fluid is released, causing a change in the relative position of the brain and intracranial contents. This is

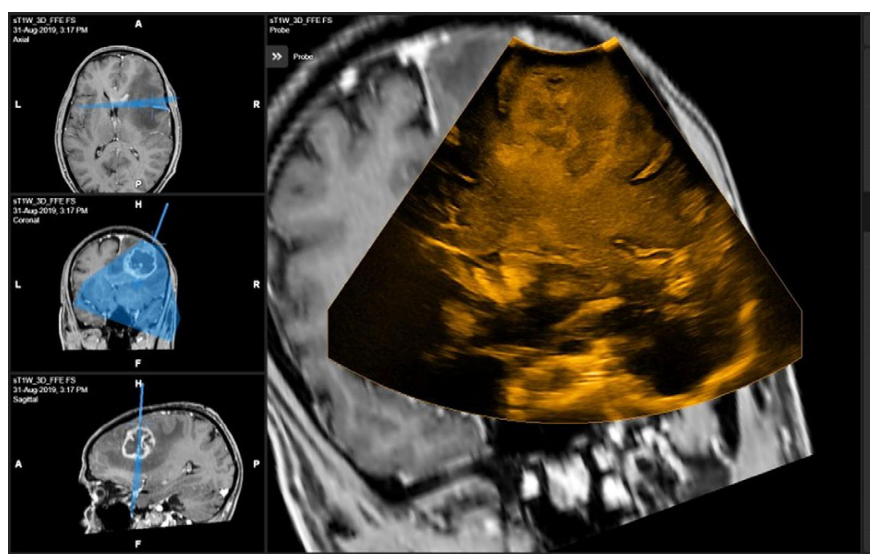

B 


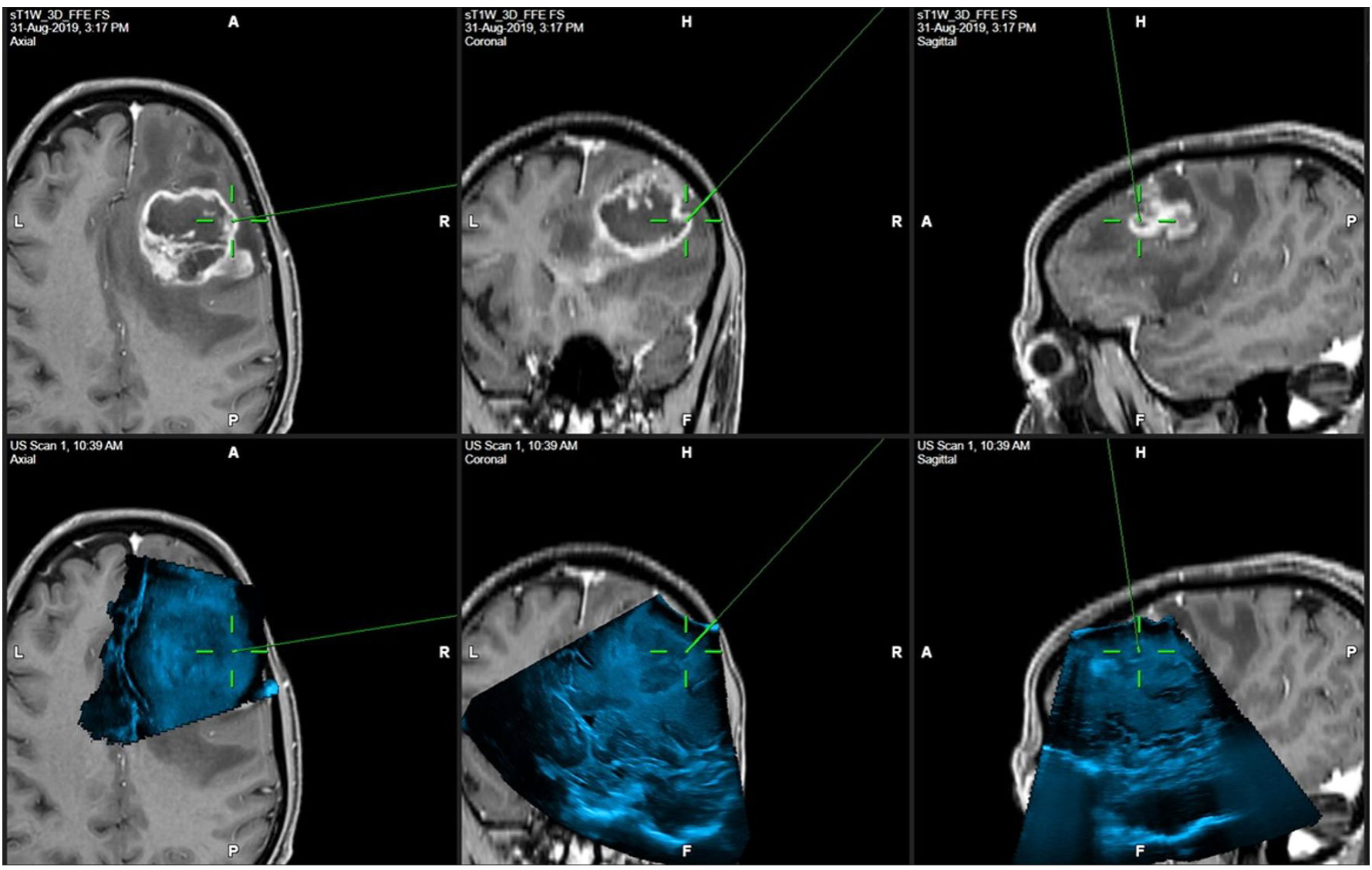

Fig. 8. Navigated three-dimensional ultrasonography (3DUS). This shows the screenshot after acquiring the 3DUS in the same case as in Fig. 7. Using the navigator tool (depicted virtually as the green line with cross-hairs), the ultrasound (US) images can now be seen in all three conventional planes (axial, coronal, and sagittal) along with the corresponding magnetic resonance images. This screen layout can be customized to multiple display formats.
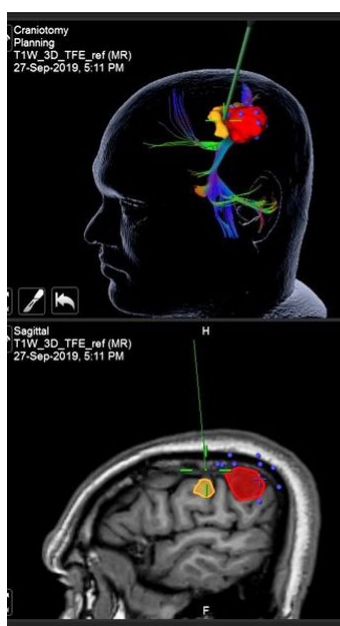

Q Q

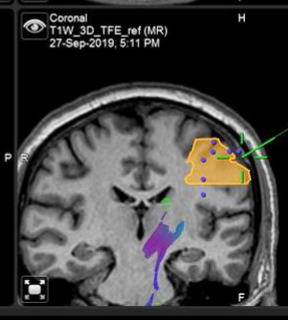

A

Fig. 9. Magnetic resonance (MR)-ultrasound (US) image fusion.

Functional information can be outlined on the MR images. A. The motor cortex (orange), corticospinal tracts (blue), and the tumor itself (red) have been segmented. B. The inferior fronto-occipital tract has been outlined in green and is co-displayed with the US images. 
the phenomenon of brain shift. Using n3DUS, brain shift can be identified and even quantified (Fig. 10). Although we do use the overlay functional MR imaging and tractography information, we rely on confirmatory intraoperative neuromonitoring using monopolar (with the patient under anaesthesia) or bipolar (with the patient awake) stimulation during surgery for all tumors near eloquent regions. Surgical resection is then conducted according to standard microneurosurgical principles. We prefer using an en bloc resection strategy for all low-grade and most high-grade intra-axial lesions. The IOUS blends seamlessly into the surgical workflow without much discomfort to either the patient (if awake) or the surgeon.

\section{Resection Control Scans}

As the resection progresses, frequent US updates are obtained. Prior to each US scan, the resection cavity is thoroughly rinsed of debris with copious amounts of saline, and hemostasis is meticulously achieved. The position of the table can be adjusted to ensure that the cavity is as vertical as possible, and that cavity is then filled with an adequate amount of saline. It is imperative to ensure that the images are of good quality and free of artifacts. Insonation is repeated in the same sequence as in the pre-resection US scans (live 2DUS sweeps followed by 3DUS acquisition if needed). Occasionally, only live 2DUS updates are obtained to acquire a rapid review of the extent of resection or to check the progress and reorient if needed (Fig. 11). Even with n2DUS, it is important to keep in mind the plane of the previously-acquired 2DUS scans to avoid either missing or mistakenly noting the presence of residue, especially when comparing non-coplanar images (Fig. 12). The challenge is to obtain images in the same plane repeatedly in cases when a very small residue needs to be detected. More importantly, once the probe is removed from the operative site, the images are no longer available for review and for use in exactly locating the residue. With n3DUS, once the navigator is brought into the field, comparative evaluation

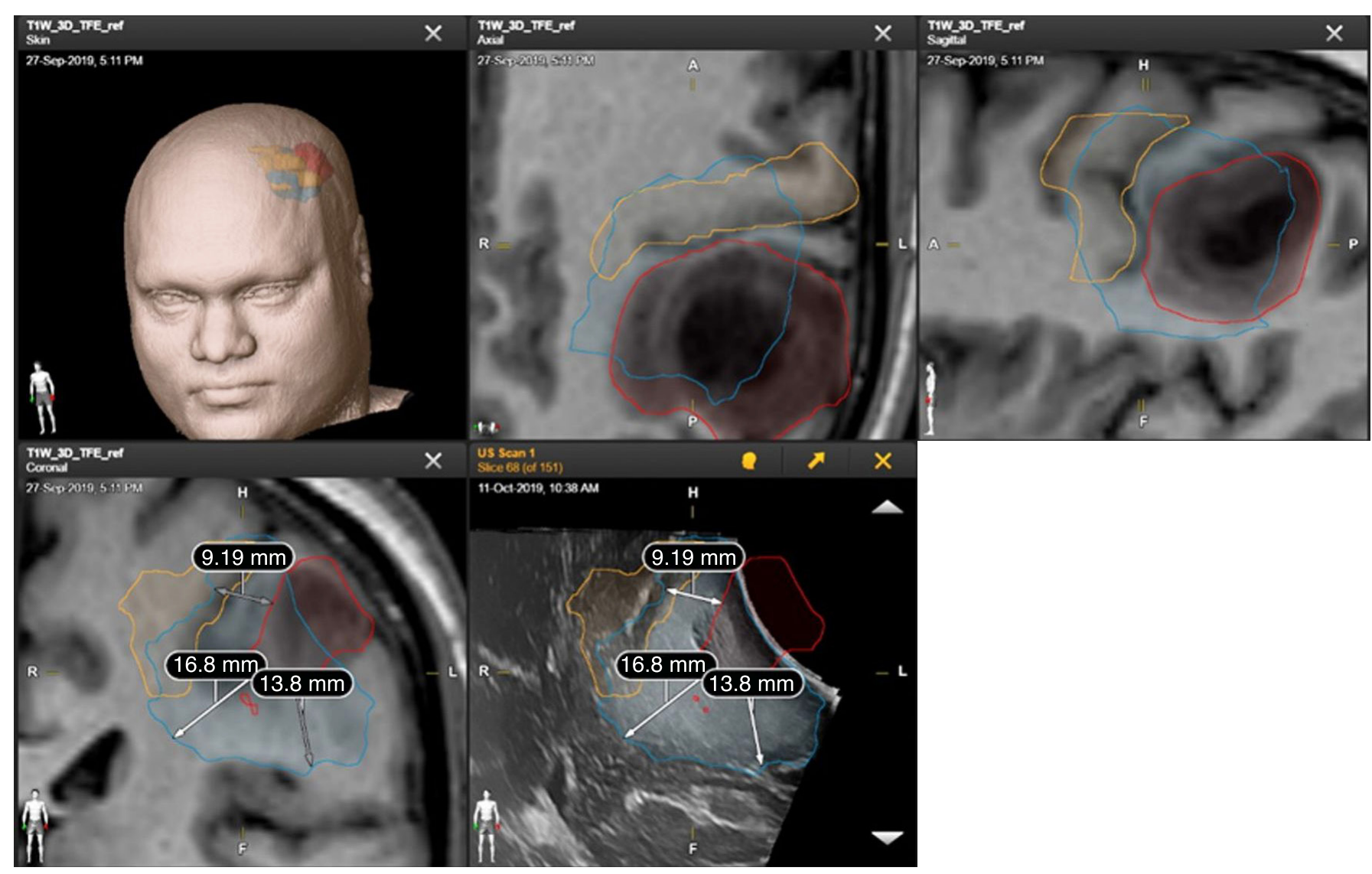

Fig. 10. Brain shift demonstrated on navigated ultrasonography. Screenshot from the navigation system shows the tumor segmented in red based on the magnetic resonance (MR) images. The motor cortex is depicted in orange. The center image of the bottom panel shows the ultrasound (US) image and the tumor, segmented here in blue, overlapping with the MR-segmented tumor (red). The shift is calculated manually in different directions and is appreciably variable in all of them. Importantly, this information can be used to infer the new position of the motor cortex even if the US cannot be used to define the motor cortex itself. 
of the serial temporally-acquired US images can be performed, and the exact location of the residue can be pinpointed and resected with confidence (Figs. 12, 13). Multiple such 3DUS scans can be repeatedly acquired as the resection progresses. Brain shift across the subsequent US scans can also be noted (Fig. 14). In addition to the conventional ACS views, the acquired images can be presented

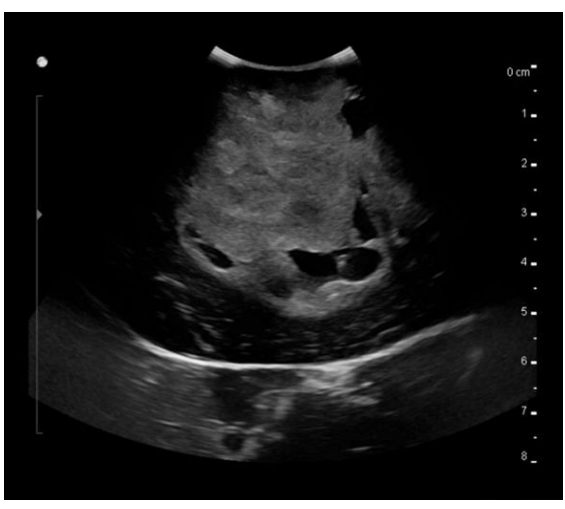

A

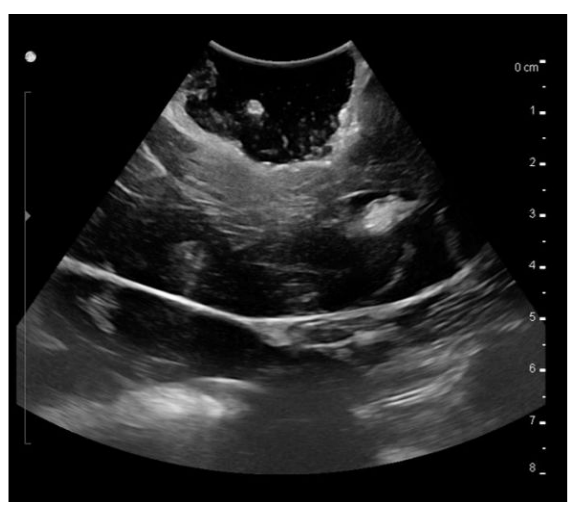

B

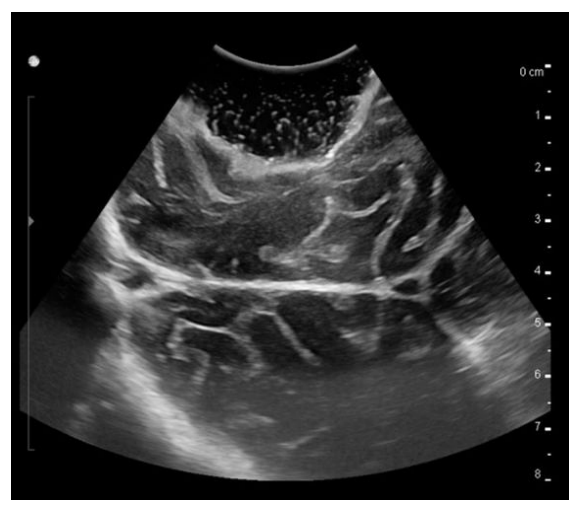

C

Fig. 11. Resection control scans using serial real-time two-dimensional (2D) grey-scale ultrasound images.

Pre-resection imaging $(A)$, intermediate imaging $(B)$, and post-resection imaging $(C)$ is seen. Note the good image resolution of the tumor mass as well as the surrounding anatomical details. However, it should also be noted that each of the images is in a different 2D plane, making direct comparison with the preceding images challenging.

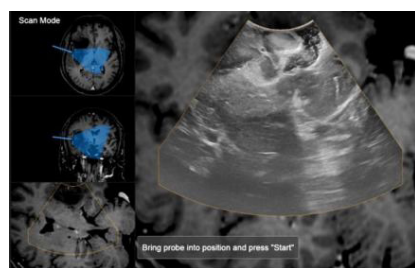

A
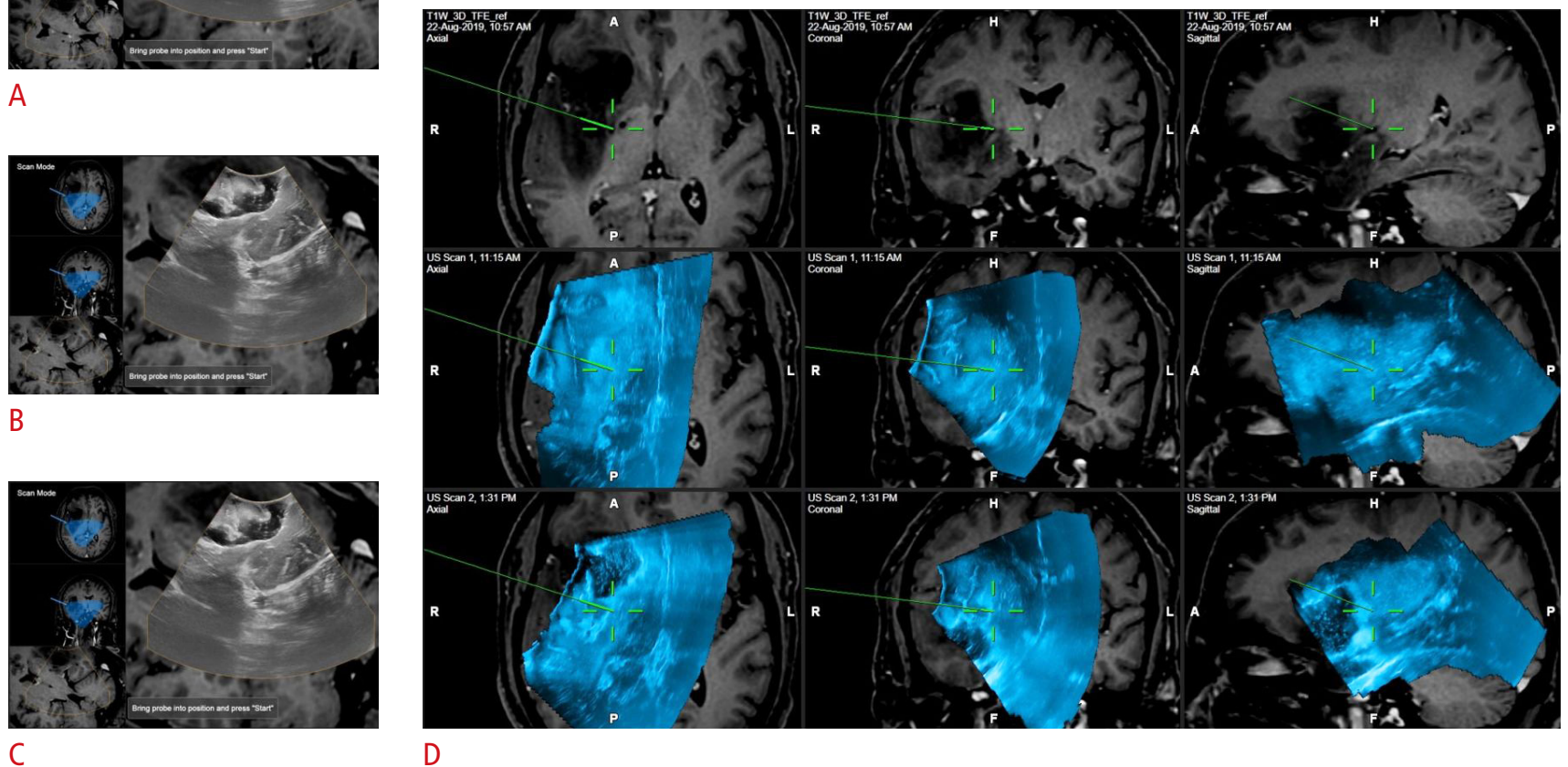

B

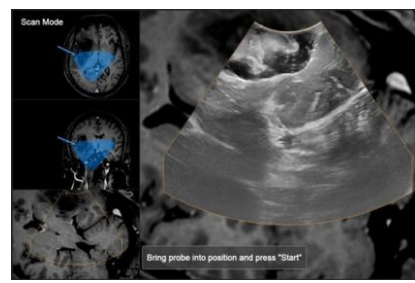

C

D

Fig. 12. Resection control scans using navigated two-dimensional ultrasonography (2DUS) versus navigated three-dimensional ultrasonography (3DUS).

A-C. The left column depicts serially-acquired navigated 2DUS images. Note the differing planes of each 2DUS image as shown in the corresponding magnetic resonance cuts. D. In the same patient, after acquiring a 3DUS scan, serial scans can be depicted side-by-side and visualized in the same planes, allowing for easier comparison. 


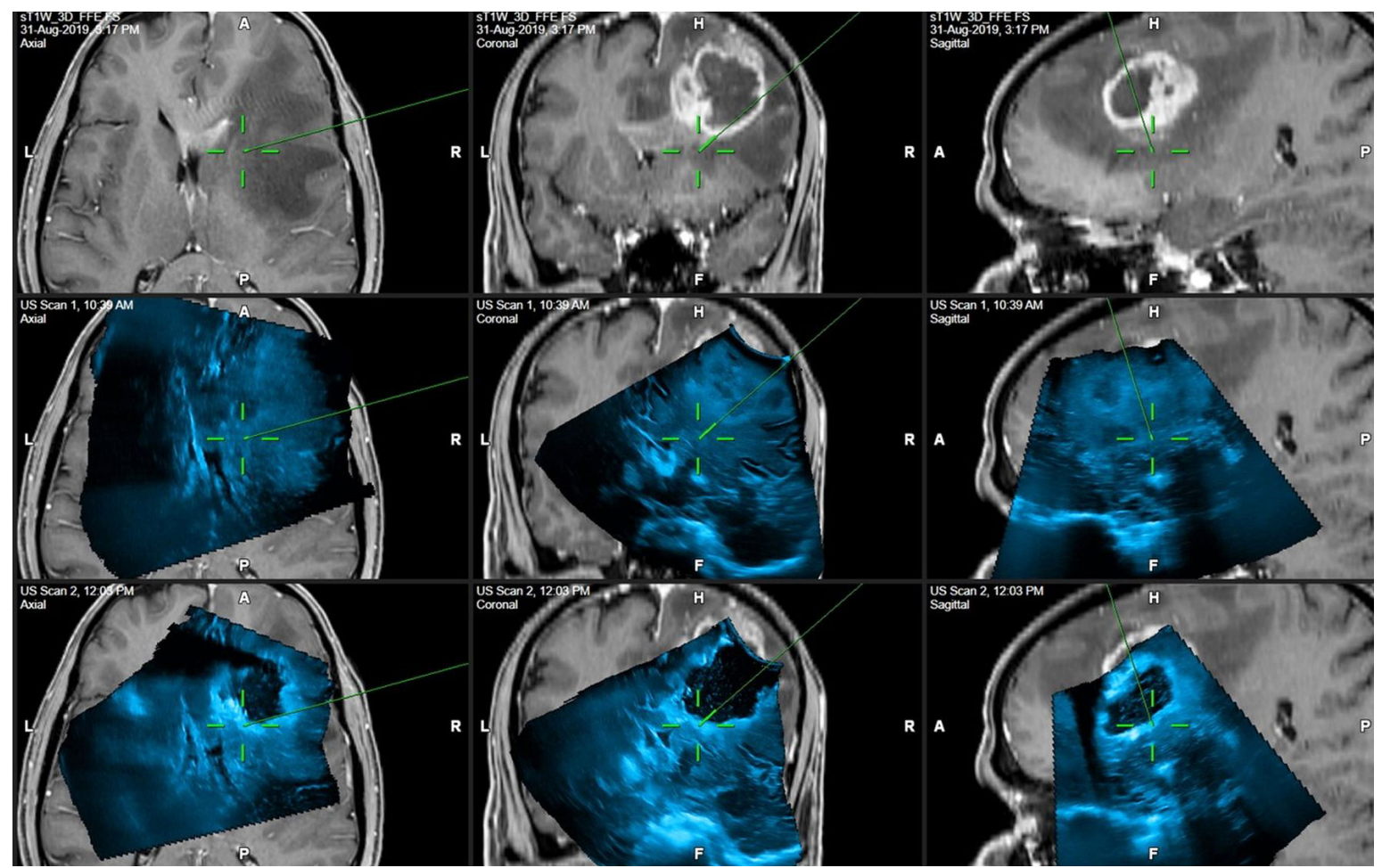

Fig. 13. Navigated three-dimensional ultrasonography showing preoperative magnetic resonance (upper row), pre-resection ultrasound (US; middle row), and post-resection US (bottom row) images.

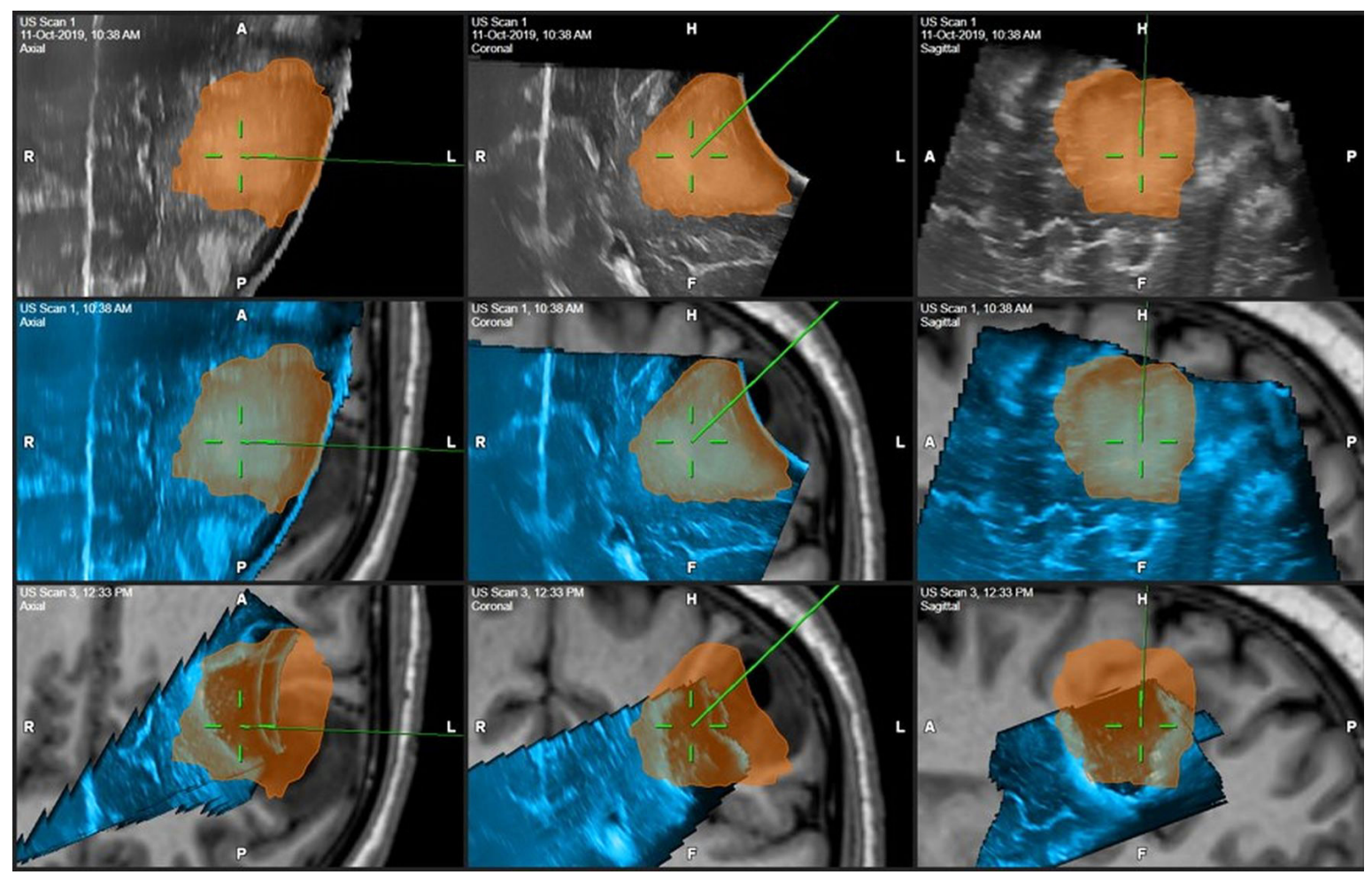

Fig. 14. Demonstration of progressive brain shift. The top row shows the pre-resection three-dimensional ultrasonography (3DUS) in the axial, coronal, and sagittal views. The tumor is outlined in orange. The middle row shows the same ultrasound (US) image superimposed on the preoperative magnetic resonance image. The brain shift is clearly visible. The bottom row shows the pre-resection US-segmented tumor and the post-resection updated 3DUS with a cavity that is displaced in all three planes with respect to the pre-resection US. 
in a more user-intuitive inline view. The inline views depict the volume in a plane parallel to the plane of the surgical view, thereby providing a more practical orientation. Combining two such mutually orthogonal views is very useful in orienting the operative cavity with respect to the acquired images (Fig. 15). Each 3DUS acquisition takes no more than 1 to 2 minutes overall, adding negligible time to the overall procedure.

\section{Discussion}

Image-guided surgery has recently become a technique of great interest, especially in brain tumors, for which the importance of the extent of resection has been well-established. One major reason for incomplete resection is the presence of residue that is missed during the conventional surgical examination of the operative cavity [4]. Navigation alone is of limited value. To facilitate resection, various intraoperative tools are available, including fluorescence, ultrasound, and MR; of the available options, IOUS is the most cost-effective [5]. Additionally, IOUS is widely available and is a versatile tool with many applications in tumor surgery $[2,3]$. By itself, 2DUS is a useful tool in the targeting and resection control of tumors. With advances in US technology, the quality of the acquired images and functionality of US systems have greatly improved. However, the issue of subjectivity and the user-dependent nature of the technique remains. The standard training of neurosurgeons does not equip them to perform and interpret US scans. Coupled with the unconventional surgical planes present during craniotomy exposures and the incomplete (lack of full-head) views on IOUS, this can cause considerable disorientation, steepening the learning curve. Furthermore, the good-quality images provided by US must be memorized by the surgeon, as once the US probe is removed from the surgical field, these images are no longer available, and the surgeon must rely on mental reconstruction to proceed with further resection. This can be challenging when chasing smaller

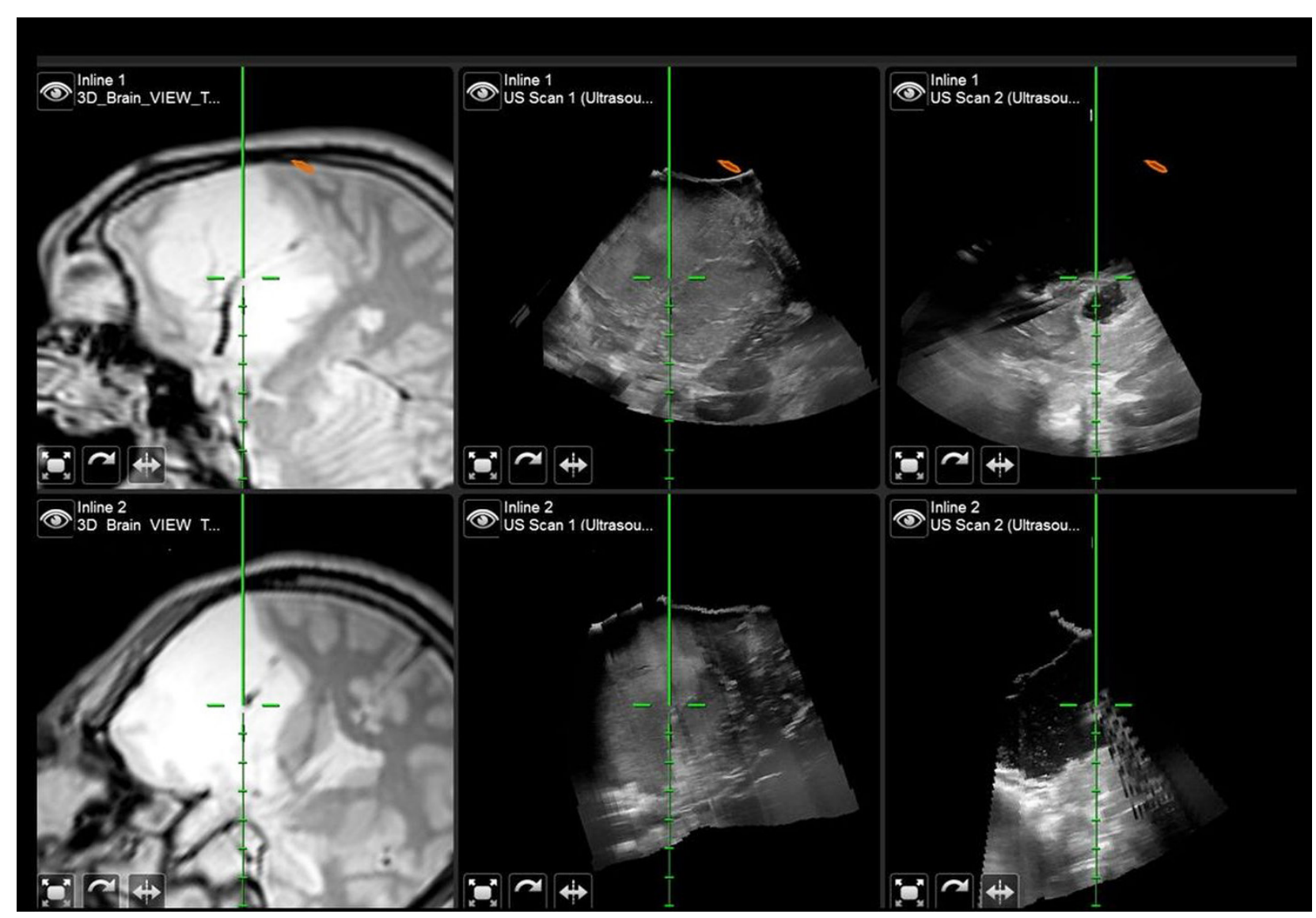

Fig. 15. Inline views of a left insular glioma. Orthogonal inline magnetic resonance views are shown in the leftmost column. The central column shows co-registered pre-resection orthogonal ultrasound (US) images, and the rightmost column displays the post-resection US images in the same orthogonal planes. The vertical green line corresponds to the direction of the surgeon's operative field of view. A tumor appearing hyperechoic on US is outlined with a white dashed line in the pre-resection (central panel) as well as post-resection (right panel) images. Note the residual tumor seen along with posterior enhancement artifacts in the right panel. 


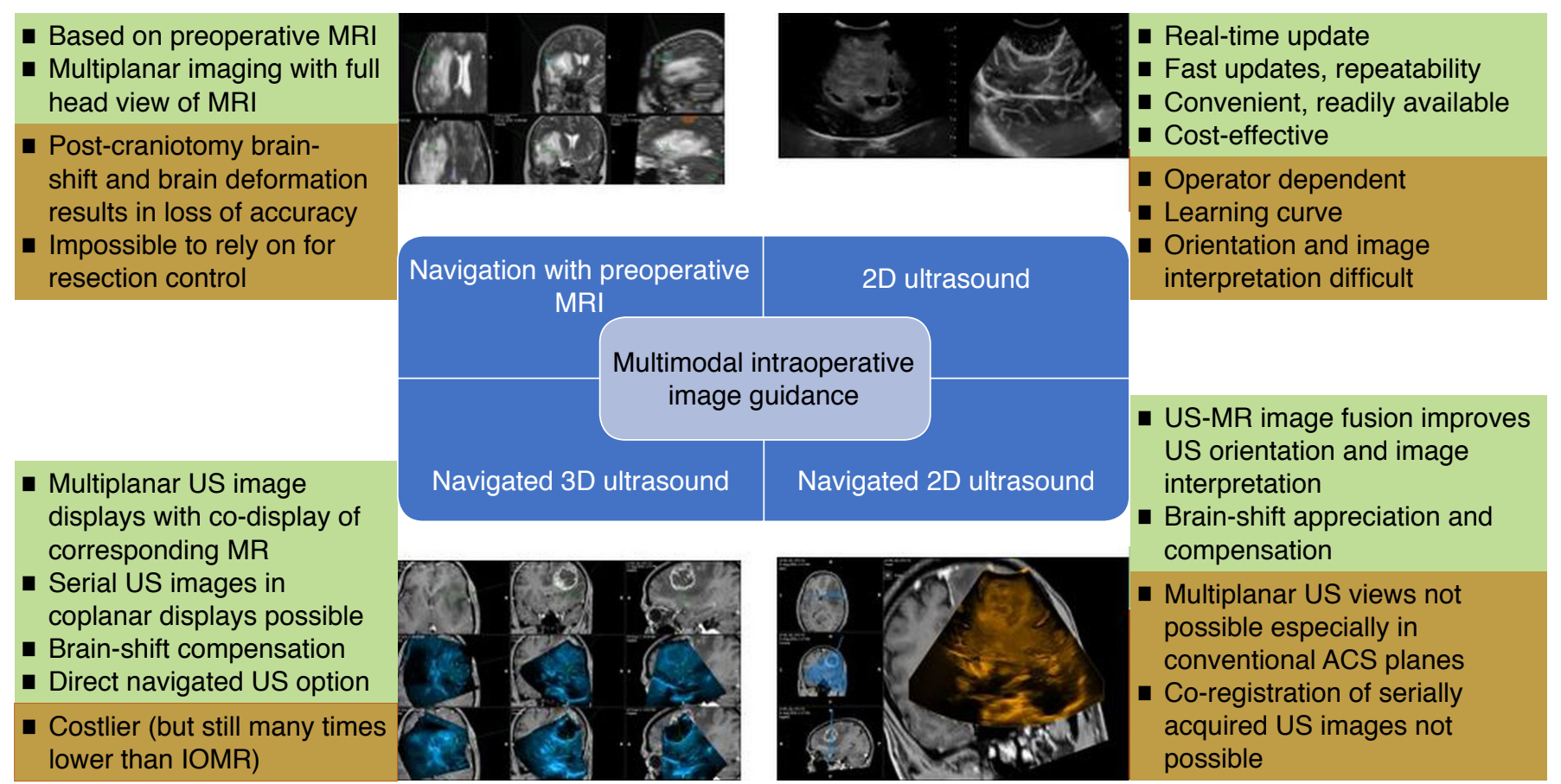

Fig. 16. Multimodal ultrasound (US)-based intraoperative image guidance. Clockwise, the US techniques improve incrementally from the top left corner (navigation) to the bottom left corner (navigated three-dimensional US). The benefits (green boxes) and limitations (orange boxes) of each of the modalities are highlighted. MRI, magnetic resonance imaging; IOMR, intraoperative magnetic resonance imaging; ACS, axial, coronal, and sagittal.

tumor remnants in the proximity of critical and eloquent structures. However, as we have demonstrated in this paper, the potential benefits of US can be unlocked using navigation and image fusion. 2DUS, n2DUS, and n3DUS have incremental benefits (Fig. 16) that build on the strengths of each component with regard to US and navigation.

Image fusion with MR allows a US image to be co-displayed with an MR image. This facilitates image interpretation, as one can use the MR image to better understand the US image as well as the overall orientation of the surgical field [6]. Tracking the US probe during the intraoperative acquisition of the US image is key to being able to navigate using the US image. Subsequently, some systems (such as that described above) can be used to acquire and reconstruct 3D volumetric US scans, which then can be reformatted in any chosen plane of representation, such as ACS views. These allow instant re-orientation of the US anatomy in the standard views, making learning easier in the initial stages of training. n3DUS also permits the serial display and comparison of multiple resection-control US scans performed sequentially, thereby allowing for the accurate assessment of small residual remnants (Figs. 8, 9). Renovanz et al. [7] showed that the sensitivity and specificity of the detection of tumor remnants were higher in nUS than in nonnUS imaging. Additional comparative studies are needed to critically analyze the benefits of nUS. Whereas most systems require the preoperative MR images to be co-displayed, some nUS systems can use solely 3DUS data for navigation, providing a direct-navigated US option. In our experience with such a system, we found this to be very convenient, especially for cases in which a preoperative MR sequence suitable for navigation is not available [8]. While utilizing such a system requires a level of proficiency in using and interpreting US images, that proficiency may be gained rather quickly when using the co-display image fusion during the early part of the learning curve. nUS can also demonstrate and compensate for brain shift (Figs. 10, 14) [6]. Though not always accurate, it is best to continue to rely on the most recently updated US image (and subsequent resection control scans) for assessing the operative field, as it represents the true status and should be relied upon for making intraoperative decisions. Basing intraoperative assessments on the updated US image with the MR image serving as a roadmap is the best approach when using nUS.

At our center, we have been using IOUS for over 12 years and nUS for almost 8 years. The initial phase of the learning curve is long, but the use of nUS can substantially shorten this phase. In our experience, IOUS is a very useful tool for the treatment of all kinds of brain tumors $[3,9]$. It is particularly useful for targeted biopsies [10] as well as the resection of intra-axial tumors [11-14]. Large 
systematic reviews and meta-analyses have also indicated that IOUS can be a very useful adjunct to improve the resections of gliomas $[15,16]$. Considering its widespread availability and relatively low cost, IOUS is a tool that should be increasingly adopted by neurosurgeons. Acquiring a good US scan remains the cornerstone of this technique and cannot be compensated for by image fusion. Moreover, post-resection artifacts are troublesome and must be minimized for accurate interpretation [17]. Despite these challenges, the learning curve can be quickly climbed with dedicated training and the frequent use of nUS. Interpretation of the post-resection US scan remains a key factor in the role of IOUS as a tool for resection control. The diagnostic accuracy of US compared to postoperative MR imaging [16] as well as pathological tumor confirmation [18] is reported to be between $60 \%$ and $100 \%$. However, comparison across studies is difficult due to the lack of uniform criteria regarding residual tumors on US and the lack of a consistent gold standard in all studies.

\section{Conclusion}

nUS is a useful addition to the neurosurgeon's intraoperative imaging armamentarium. It combines the benefits of real-time US imaging with powerful navigation technology and is a versatile and multifunctional adjunct. In the hands of an operator with experience in US image acquisition and interpretation, it can potentially be a useful tool for the surgical treatment of brain tumors.

ORCID: Aliasgar Moiyadi: https://orcid.org/0000-0002-4082-2004

\section{Author Contributions}

Conceptualization: Moiyadi A. Data acquisition: Moiyadi A, Shetty P, Singh V, Yeole U, Mishra A, Shaikh S. Data analysis or interpretation: Yeole $U$, Moiyadi A. Drafting of the manuscript: Yeole U, Moiyadi A. Critical revision of the manuscript: Yeole U, Singh V, Mishra A, Shaikh S, Shetty P, Moiyadi A. Approval of the final version of the manuscript: all authors.

\section{Conflict of Interest}

No potential conflict of interest relevant to this article was reported.

\section{Supplementary Material}

Supplementary Fig. 1. High-resolution image of Fig. 4A (https://doi. org/10.14366/usg.20044).

Supplementary Fig. 2. High-resolution image of Fig. 4B (https://doi. org/10.14366/usg.20044).
Supplementary Data 1. Technical details and workflow (https://doi. org/10.14366/usg.20044).

\section{References}

1. Nabavi A, Black PM, Gering DT, Westin CF, Mehta V, Pergolizzi RS $\mathrm{Jr}$, et al. Serial intraoperative magnetic resonance imaging of brain shift. Neurosurgery 2001;48:787-797.

2. Moiyadi AV. Objective assessment of intraoperative ultrasound in brain tumors. Acta Neurochir (Wien) 2014;156:703-704.

3. Moiyadi A, Shetty P. Objective assessment of utility of intraoperative ultrasound in resection of central nervous system tumors: a costeffective tool for intraoperative navigation in neurosurgery. J Neurosci Rural Pract 2011;2:4-11.

4. Orringer D, Lau D, Khatri S, Zamora-Berridi GJ, Zhang K, Wu C, et al. Extent of resection in patients with glioblastoma: limiting factors, perception of resectability, and effect on survival. J Neurosurg 2012;117:851-859.

5. Eljamel MS, Mahboob SO. The effectiveness and cost-effectiveness of intraoperative imaging in high-grade glioma resection; a comparative review of intraoperative ALA, fluorescein, ultrasound and MRI. Photodiagnosis Photodyn Ther 2016;16:35-43.

6. Prada F, Del Bene M, Mattei L, Casali C, Filippini A, Legnani F, et al. Fusion imaging for intra-operative ultrasound-based navigation in neurosurgery. J Ultrasound 2014;17:243-251.

7. Renovanz M, Hickmann AK, Henkel C, Nadji-Ohl M, Hopf NJ. Navigated versus non-navigated intraoperative ultrasound: is there any impact on the extent of resection of high-grade gliomas? A retrospective clinical analysis. J Neurol Surg A Cent Eur Neurosurg 2014;75:224-230

8. Moiyadi AV, Shetty P. Direct navigated 3D ultrasound for resection of brain tumors: a useful tool for intraoperative image guidance. Neurosurg Focus 2016;40:E5.

9. Moiyadi AV. Intraoperative ultrasound technology in neuro-oncology practice: current role and future applications. World Neurosurg 2016;93:81-93.

10. Patil AD, Singh V, Sukumar V, Shetty PM, Moiyadi AV. Comparison of outcomes of free-hand 2-dimensional ultrasound-guided versus navigated 3-dimensional ultrasound-guided biopsy for supratentorial tumours: a single-institution experience with 125 cases. Ultrasonography 2019;38:255-263.

11. Moiyadi AV, Shetty P, John R. Non-enhancing gliomas: does intraoperative ultrasonography improve resections? Ultrasonography 2019;38:156-165.

12. Moiyadi AV, Shetty PM, Mahajan A, Udare A, Sridhar E. Usefulness of three-dimensional navigable intraoperative ultrasound in resection of brain tumors with a special emphasis on malignant gliomas. Acta Neurochir (Wien) 2013;155:2217-2225. 
13. Moiyadi AV, Kannan S, Shetty P. Navigated intraoperative ultrasound for resection of gliomas: predictive value, influence on resection and survival. Neurol India 2015;63:727-735.

14. Dubey S, Janu A, Chaudhari S, Moiyadi A. Navigable 3D-ultrasound facilitates supra-radical resections beyond the contrast-enhancing boundaries in malignant gliomas. J Neurol Surg A Cent Eur Neurosurg 2016;77:372-375.

15. Mahboob S, McPhillips R, Qiu Z, Jiang Y, Meggs C, Schiavone $G$, et al. Intraoperative ultrasound-guided resection of gliomas: a meta-analysis and review of the literature. World Neurosurg 2016:92:255-263.

16. Trevisi G, Barbone P, Treglia G, Mattoli MV, Mangiola A. Reliability of intraoperative ultrasound in detecting tumor residual after brain diffuse glioma surgery: a systematic review and meta-analysis. Neurosurg Rev 2019 Aug 14 [Epub]. https://doi.org/10.1007/ s10143-019-01160-x.

17. Selbekk T, Jakola AS, Solheim 0 , Johansen TF, Lindseth F, Reinertsen I, et al. Ultrasound imaging in neurosurgery: approaches to minimize surgically induced image artefacts for improved resection control. Acta Neurochir (Wien) 2013;155:973-980.

18. Zhang G, Li Z, Si D, Shen L. Diagnostic ability of intraoperative ultrasound for identifying tumor residual in glioma surgery operation. Oncotarget 2017;8:73105-73114. 